\title{
The Homogeneity Research of Urban Rail Transit Network Performance
}

\author{
Wang Fu-jian and Ruan Shu-bin \\ College of Civil Engineering and Architecture, Zhejiang University, Hangzhou 310058, China
}

\begin{abstract}
Urban Rail Transit is an important part of the public transit, it is necessary to carry out the corresponding network function analysis. Previous studies mainly about network performance analysis of a single city rail transit, lacking of horizontal comparison between the multi-city, it is difficult to find inner unity of different Urban Rail Transit network functions. Taking into account the Urban Rail Transit network is a typical complex networks, so this paper proposes the application of complex network theory to research the homogeneity of Urban Rail Transit network performance. This paper selects rail networks of Beijing, Shanghai, Guangzhou as calculation case, gave them a complex network mapping through the L, P Space method and had a static topological analysis using complex network theory, Network characteristics in three cities were calculated and analyzed form node degree distribution and node connection preference. Finally, this paper studied the network efficiency changes of Urban Rail Transit system under different attack mode. The results showed that, although rail transport network size, model construction and construction planning of the three cities are different, but their network performance in many aspects showed high homogeneity.
\end{abstract}

\section{Introduction}

Urban Rail Transit does not occupy ground road resources, large capacity, fast and convenient features, ease urban traffic congestion to a great extent, so the major cities in China have been increased investment in rail transportation. The total length of rail transit lines in operation by the $621 \mathrm{~km}$ (2006) soared to 2, $408 \mathrm{~km}$ (2013), the respective rail vehicle operators also increased to 14,366 units from the 2, 764 units [1]. Beijing, Shanghai, Guangzhou has basically formed a stable operation of the rail transit network, Hangzhou, Nanjing, Wuhan, Xi'an, Qingdao also in actively construction of rail transportation, Urban Rail Transit will become an important part of the public transit. In order to ensure efficient and safe operation of the urban rail system, it is necessary to make the corresponding network performance analysis for Urban Rail Transit system.

Urban Rail Transit network is a typical complex network of Nodes and Links composition [2]. With the maturation of complex network theory, more and more domestic and foreign scholars with their research to analyze the performance of Urban Rail Transit network, mainly including the prevention of topological analysis, node dynamics, network attacks and subsequent failure of the network Chaos and synchronization of complex networks analysis. As Latora et al [3] found a smallworld characteristics of Boston subway system, and the corresponding mathematical modeling; Angeloudis et al [4] studied the overall efficiency of the rail network changes situation under different network nodes attack mode; Derrible et al [5] conducted a thorough analysis of the Urban Rail Transit scale-free and small world, and studied the relationship between transfer hub station and network robustness; Rui et al [6] conducted a simulation of complex network evolution Research for Kuala Lumpur Metro. Yang et al [7] studied the dynamic characteristics of urban rail network performance under cyber attack using complex network theory; Liu et al[8] conducted a complex topology modeling and the appropriate network parameters analysis for Wuhan subway network using the L, P Space method; Zhang et al[9] studied abroad metro reliability and robustness on the basis of the system analysis for complex network parameters.

Previous studies focused on analysis of network performance parameters of Urban Rail Transit, less studied the homogeneity of Urban Rail Transit network performance in different cities. The so-called homogeneous, referring to the inherent unity of the network topology properties. That is different network topologies have the same or similar network performance. Through horizontal comparison analysis of Urban Rail Transit network performance in Beijing, Shanghai and Guangzhou, it was found to have a high degree of homogeneity in the topology and network performance, provide an effective reference to expand research the urban rail transit in other cities.

\section{Network construction of Urban Rail Transit system}


Urban Rail Transit system is a complex network having a defined topological relations, The Urban Rail Transit system network methods include the R Space method and the L, P Space method. The R Space method is to track the rail-line as a complex network node, and the site of line intersects as a complex network link, the number of the site what two lines intersecting as a complex network connection weights, to construct the $\mathrm{R}$ spatial network model. In contrast, the L, P Space method is to track the site as a complex network node, and the rail-line as the complex network link, so this method is a direct mapping to track network. the configuration differences of the L,P Space method as follows:

(1). The L space network model. The network model is a direct mapping of the rail network diagram that if two adjacent sites in a track circuit, then connect it with the link.

(2). The P space network model. When two sites are connected by at least one rail line, then connect it with the link. i.e. Line $=\left(p_{1}, p_{2}, p_{3} \ldots p_{n}\right)$, the site $p_{1}$ and the sites $p_{2}, p_{3} \ldots p_{n}$ are to establish a connection.

Fig. 1 shows the display method of the L, P Spcce.

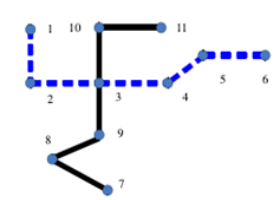

(a) L Space

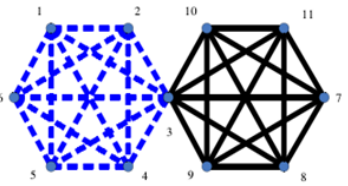

(b) P Space

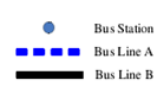

(c) Icon
Figure 1. The display method of the L, P Spcce.

This paper selected the L, P spatial network method for urban rail network mapping. The L space network model is applied to study the degree distribution and network efficiency of the track network. The $\mathrm{P}$ space network model is suitable for the study of transfer parameters. The above two methods have their limitations respectively, so this paper will combine the use of these two methods.

Taking into account the Urban Rail Transit system in Beijing, Shanghai, Guangzhou developed more perfect and mature, so this paper make the Urban Rail Transit networks of above three cities as the study object. Urban Rail Transit network data including line network operators table, name of the site, ID number of the site, name of the line, station location (latitude and longitude) and other items, All data items got through the Baidu maps API interface. Convert the data file for the site ID retrieval matrix, site latitude and longitude matrix, adjacency matrix and a series of data basis for complex network analysis based on the above L, P spatial network method.

\section{Topology homogeneity of urban rail transport network}

\subsection{Static topological features}

Static topological characteristics of complex networks including degree and degree distribution, the network diameter and the average path length, betweenness and clustering coefficient.

(1). Degree and degree distribution. A degree of the node $\mathrm{i}$ is the number of edges associated with the node $i$. The average degree value of each node is the degree of the entire network, i.e.

$$
<k>=\overline{k_{i}}=\left(\sum_{i \in N} k_{i}\right) / N
$$

$p(k)$ represents the probability of each node degree distribution.

(2). The network diameter and the average path length. In the complex network theory, define the distance between nodes $n_{i}, n_{j}$ is the number of edges in the shortest path connecting nodes $n_{i}, n_{j}$. The network diameter $\mathrm{D}$ is the maximum distance between any two nodes in the network, i.e.

$$
D=\max _{i, j}\left(d_{i j}\right)
$$

The average path length of the network is the average distance between all nodes.

(3). Betweenness. Betweenness divided the edges betweenness and the node betweenness. The so-called edge betweenness, refers to the network between all nodes on the shortest path through which the ratio of the edge, the node betweenness and the edge betweenness have the similar meaning.

(4). Clustering coefficient. clustering coefficient reflects the degree of aggregation of nodes in the network, the degree of node $n_{i}$ is $k_{i}$, i.e. adjacent to the surrounding $k_{i}$ nodes. If the interconnectivity between these $k_{i}$ nodes, even up to the $k_{i}\left(k_{i}-1\right) / 2$ links, Suppose even number of links it actually exists is $n_{k_{i}}$, then the node clustering coefficient $C_{i}$ is:

$$
C_{i}=n_{k_{i}} /\left(k_{i} \times\left(k_{i}-1\right)\right)
$$

Network clustering coefficient $\mathrm{C}$ is the arithmetic mean of each node clustering coefficient:

$$
C=\frac{1}{N} \sum_{i=1}^{N} C_{i}
$$

\subsection{Homogeneous topology}

Through computer programming to Beijing, Shanghai and Guangzhou Urban Rail Transit network topology packet characteristic calculation, the specific results are shown in Table 1.

By the Table 1, it can be seen, Urban Rail Transit network scale of the three cities, but the average degree of nodes, the network diameter, the average path length, the average clustering coefficient roughly equivalent, It illustrates the complex network topology homogeneity of the three cities. 
Table 1. Topology eigenvalues of the Urban Rail Transit network

\begin{tabular}{c|ccccccc}
\hline Space & City & Nodes & Average degree & $\begin{array}{c}\text { Network } \\
\text { diameter }\end{array}$ & $\begin{array}{c}\text { Average path } \\
\text { length }\end{array}$ & $\begin{array}{c}\text { Average } \\
\text { Betweenness }\end{array}$ & $\begin{array}{c}\text { Average clustering } \\
\text { coefficient }\end{array}$ \\
\hline \multirow{3}{*}{ Space L } & Beijing & 275 & 2.24 & 45 & 15.0662 & 2309.03 & 0.0024 \\
& Shanghai & 291 & 2.24 & 41 & 15.1194 & 2445.5 & 0.0029 \\
& Guangzhou & 144 & 2.17 & 44 & 12.8969 & 962.2 & 0 \\
\hline \multirow{3}{*}{ Space P } & Beijing & 275 & 26.61 & 5 & 2.5349 & 543.9236 & 0.9231 \\
& Shanghai & 291 & 29.52 & 4 & 2.2184 & 469.2096 & 0.9127 \\
& Guangzhou & 144 & 19.47 & 4 & 2.3344 & 168.9444 & 0.928 \\
\hline
\end{tabular}

L spatial clustering coefficient of the three cities substantially 0 , which is determined by the properties of the construction of urban rail transit systems, expensive urban rail construction costs and determine the sparsity of oligomeric class line network, Most sites track just two sites adjacent to and substantially on the same line, so the clustering coefficient is 0 . In contrast spatial clustering coefficient of the P Space, then it was higher than 0.91, indicating clustering between different lines are relatively strong, Urban Rail Transit network transfer functions are strongly reflected and the average path length of the $\mathrm{P}$ Space network also corroborated this judgment. It should be noted here. The transfer here refers to the transfer of different urban rail transit lines, and the transfer between urban rail transit sites and other means of transport (such as cars, buses, etc.) is not included. that can see, the average path length of the Space P network to Beijing, Shanghai, Guangzhou, respectively 2.5349, 2.2184, 2.3344. Adjacency matrix DP of the Space P network reveals transfer relationship about urban rail complex network, processing to obtain the distance matrix of the Space $\mathrm{P}$ network using the shortest path algorithm, i.e. PDist. $\operatorname{PDist}(i, j)-1$ represents the number of transfers site $i$ to the site $j$, and if we can get the site of the travel demand distribution $S_{i, j}$, that can calculate the average number of transfers to the entire urban rail complex networks, i.e. Aver_P:

$$
\text { Aver_P }=\frac{\left(\sum_{i}^{m} \sum_{j}^{n}\left(S_{i, j} *(\operatorname{PDist}(i, j)-1)\right)\right)}{\sum_{i}^{m} \sum_{j}^{n} S_{i, j}}
$$

Aver_P reflects the level of transfers to the entire urban rail complex networks, the smaller the value indicates that the better direct accessibility of the system and the higher the efficiency of the system. When the demand for travel distribution rail network between sites is uniform distribution function, The average transfer times of rail transit network of the three cities, i.e. Aver_P, is $1.5349,1.2184,1.3344$, It showed that the number of rail lines network, topology, the number of transfer hubs of the three cities are different, but have a high homogeneity in the transfer performance.

\subsection{Uniformity of distribution}

Respectively, performed the function fitting to the site degree probability distribution of Beijing, Shanghai, Guangzhou's Urban Rail Transit network, the results shown in Fig. 2.

Fitting functions are:

$$
\begin{aligned}
& \text { Beijing: } P(K)=0.04000+\frac{1.02329 \times e^{-2 \times\left(\frac{K-1.69619}{0.45999}\right)^{2}}}{0.45999 \times \sqrt{\pi}} \\
& \text { Shanghai: } P(K)=0.01947+\frac{0.75825 \times e^{-2 \times\left(\frac{K-1.91034}{0.77438}\right)^{2}}}{0.77438 \times \sqrt{\pi}} \\
& \text { Guangzhou: } P(K)=0.03241+\frac{0.73099 \times e^{-2 \times\left(\frac{K-1.89232}{0.74341}\right)^{2}}}{0.74341 \times \sqrt{\pi}}
\end{aligned}
$$

where: $\mathrm{K}$ is the site degree, and $p(k)$ represents the probability of each node degree distribution. Specific topology eigenvalues of the Urban Rail Transit network Can refer to Table 1.

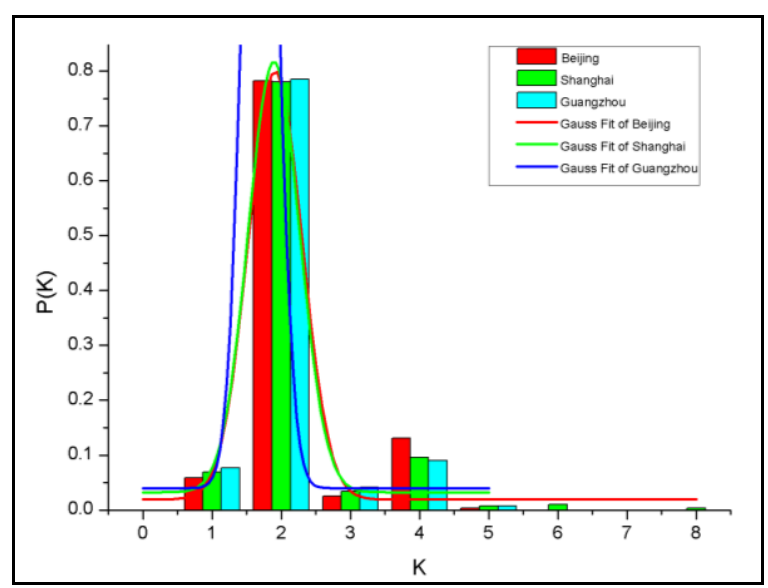

Figure 2. Degree distribution fitting (Space L).

The rail transit network degree distribution fitting function of the three cities was Gaussian distribution, between poisson distribution (ER random network and the small-world networks) and Power laws distribution (BA scale-free network) between, While the average degree of the Space L,P are small,, indicating good smallworld characteristics, it was found that three urban rail transport network are random scale-free networks. Although, the construction planning and the construction model of different cities' rail transit network are different, with a high degree of unity which generates the type of 
network topology, indicating the internal homogeneity of the urban rail network.

\subsection{Assortative coefficient of the Urban Rail Transit network}

Assortative coefficient of the complex network describes the connection between nodes preferences, indirectly reflects the evolution of the network organization strategy. If relatively large degree nodes tend to connect relatively large degree nodes, Then the network is the degree - the degree of positive correlation, it is also called Assortativeness. Newman [10] use the Pearson correlation coefficient (r) of edge nodes at both ends to describe the degree - the degree of correlation.

Pearson correlation coefficient of node degrees, also known as the Assortativity coefficient that defined as:

$$
r=\frac{M^{-1} \sum_{e_{i j} \in E} k_{i} k_{j}-\left[M^{-1} \sum_{e_{i j} \in E} \frac{1}{2}\left(k_{i}+k_{j}\right)\right]^{2}}{M^{-1} \sum_{e_{i j} \in E} \frac{1}{2}\left(k_{i}^{2}+k_{j}^{2}\right)-\left[M^{-1} \sum_{e_{i j} \in E} \frac{1}{2}\left(k_{i}+k_{j}\right)\right]^{2}}
$$

where: respectively, $k_{i}, k_{j}$ is the degrees of the two nodes $v_{i}, v_{j}$ to link $e_{i j} ; \mathrm{M}$ is the total number of network links; $\mathrm{E}$ is the set of links of the network. The range for the Pearson correlation coefficient(r) is $0 \leq|r| \leq 1$. When $\mathrm{r}<0$, the network is negative, i.e. NoAssortativeness; when $r>0$, the network is positively, i.e. Assortativeness; When $\mathrm{r}=0$, the network is irrelevant.

Processing the urban rail network adjacency matrix of Beijing, Shanghai, Guangzhou, to give the Pearson correlation coefficient were $0.0198,0.0190,0.0827$. The results illustrated the rail network of the three cities are assortative network, i.e. relatively large degree nodes tend to connect relatively large degree nodes. Since the rail network is sparse network and the high cost of construction, the new lines will always want to be able to connect the hub sites which have larger degree together, and to radiate more areas. With well-developed network structure, the new lines will be more emphasis on the connection between the suburb sites of the network which have smaller degree, the pearson correlation coefficient of Beijing, Shanghai was about 0.02, while Guangzhou was 0.08 , had higher assortative coefficient than Beijing and Shanghai. Shows that Urban Rail Transit network of Beijing and Shanghai has been the better development, the Urban Rail Transit network of Guangzhou is still in the growth evolution period.

\section{Functionality homogeneity of rail network}

With people's increasing reliance on the urban rail network, Analysis of functionality and reliability for urban rail networks are also becoming more important. Urban rail network failures include internal faults and external faults, Internal fault system mainly refers to the aging of internal disturbance fault itself generated, External fault mainly refers to the network fault external factors such as natural disasters, terrorist attacks, traffic congestion. External fault including the random attack, the maximum degree attack, the maximum betweenness attack from the attack mode[11]. Since the terrorist attacks and other malicious attacks against the subway station mainly, so fragile and reliability studied in this paper is about the node attack of the urban rail network.

\subsection{Attack mode}

Specific steps of the three kinds of network nodes attack mode are as follows:

(1). The random attack. Initially, randomly select a node in the network to delete, update the network adjacency matrix, update network topology parameters. Repeated the operation, until all the nodes are removed from the network.

(2). The maximum degree attack. Initially, compute node degree of the network, randomly select a node form a set of nodes which have the same maximum node to delete, update the network topology parameters. constantly repeating the above operations until all nodes are removed from the network.

(3). The maximum betweenness attack. Similar to the maximum degree attack, but the attack node selection rule becomes the maximum betweenness nodes.

\subsection{Network efficiency analysis}

To measure changes in urban rail network performance under attack, network efficiency herein chosen to measure the performance of the network topology.

Defined the node efficiency between nodes $n_{i}, n_{j}$ as $\tau_{i j}$, i.e.

$$
\tau_{i j}=1 / d_{i j}
$$

$d_{i j}$ is the shortest path between network nodes. According to the complex network theory, the average efficiency of all nodes on the network is the global efficiency of the network, i.e. $E_{g l o b}$ :

$$
E_{g l o b}=\left(2 \sum_{i<j \in N} \frac{1}{d_{i j}}\right) /(N(N-1))
$$

where: $d_{i j}$ is the shortest path between network nodes; $\mathrm{N}$ is the number of nodes of entir network.

Fig. 3-Fig. 5 are schematic network efficiency changes of Beijing, Shanghai, Guangzhou rail transit network under the random attack, the maximum degree attack and the maximum betweenness attack. As can be seen, variation rail network efficiency of the three cities are common under the above three attack modes-showing "waterfall" down. Among them, the maximum degree attack and the maximum betweenness attack have 
the quite same destroying effect on the Urban Rail Transit network, they were significantly higher than the random attacks.

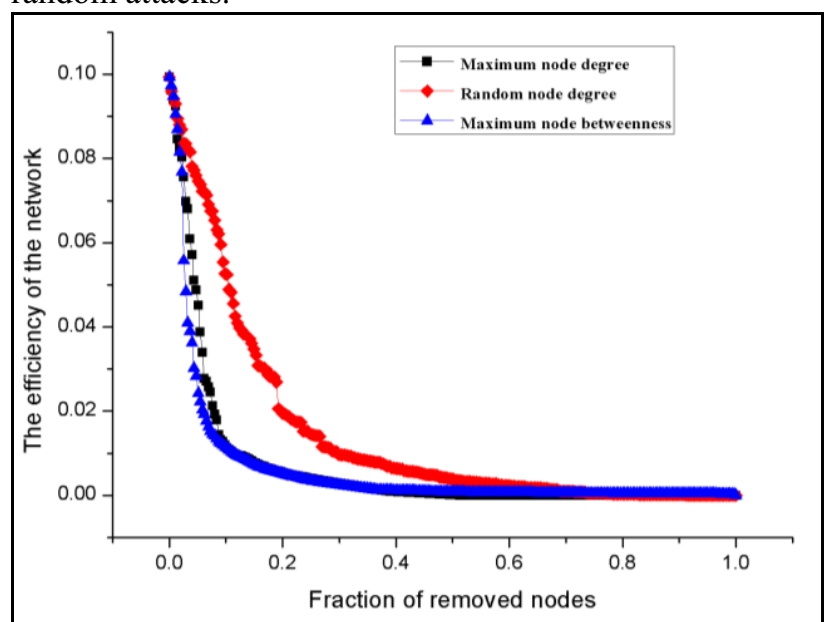

Figure 3. Network efficiency changes of Beijing urban rail network.

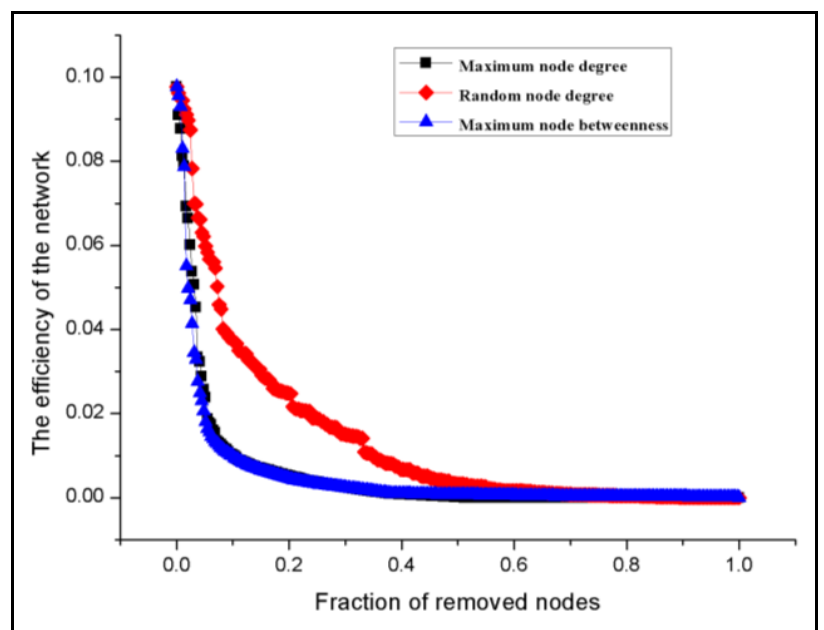

Figure 4. Network efficiency changes of Shanghai urban rail network.

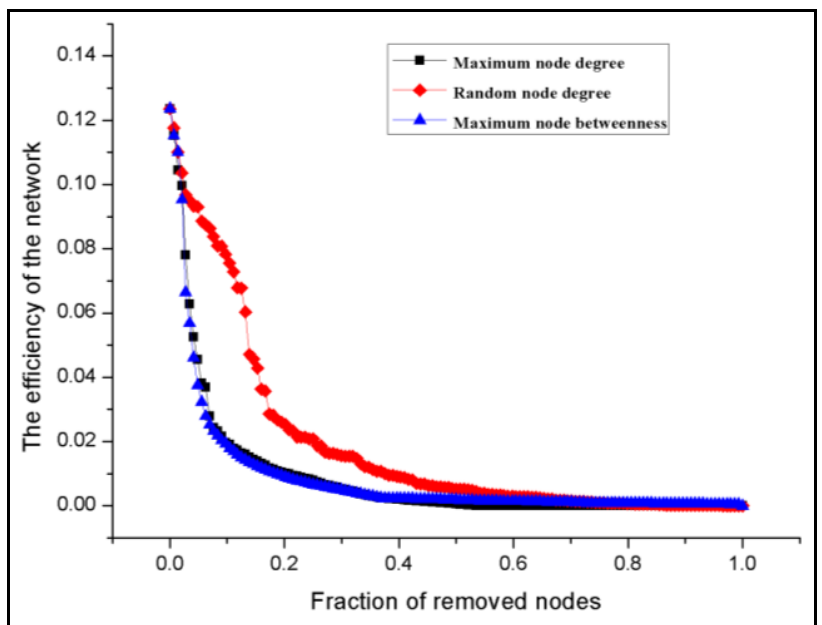

Figure 5. Network efficiency changes of Guangzhou urban rail network.

Under the maximum degree attack and the maximum betweenness attack, removed only $8 \%$ of the network nodes, it makes Beijing rail network efficiency decreased by $80 \%$ and $85 \%$, Shanghai and Guangzhou are similar. It shows that in the early attacks, a single node removal can cause a lot of network failure. And under the random attack mode, "waterfall" down still showed more obvious, just a little gentle slope only, This is due to the degree and degree distribution of the Urban Rail Transit network. The urban rail network are random scale-free networks, only a few high degree nodes, the degree most nodes are 2 , so that performed homogeneity under the three attack modes, i.e. the random attack is robust, but the network performed extremely fragile under the maximum degree attack and the maximum betweenness attack.

\subsection{Analysis of the functional homogeneity}

This paper make the horizontal comparison on the efficiency of the rail network in the three cities, Fig.6 shows the changes in network efficiency of the Urban Rail Transit in the three cities under the maximum degree attack. Note that because the network nodes and the initial value of efficiency are different, in order to perform horizontal comparison, this paper was carried out normalization process-- The abscissa is the ratio of a node is removed, the vertical axis is the percentage of network efficiency. As can be seen, although the Guangzhou rail transit network nodes far less than Beijing and Shanghai, but the robustness of its network is strongest, Guangzhou has the best description of the network topology in the three cities structure.

At the same time it can be seen from the Fig.6, when the ratio of removed nodes reaches a "threshold", Urban Rail Transit network efficiency will drop to zero. By accessing the relevant parameters matrix of the program, specific values can be obtained as shown in Table 2 . From Table 2, when fraction of removed nodes is in the range of 0.51273 to 0.51636 , The efficiency of the Beijing Urban Rail Transit network will drop to zero, the fraction range of Shanghai is 0.51203 to 0.51546 , and Guangzhou is 0.53472 to 0.54167 , so the threshold can be weighed as 0.52 , Further illustrates the homogeneity of the Urban Rail Transit network topology in the three cities, also provide an effective reference to expand research the urban rail transit in other cities.

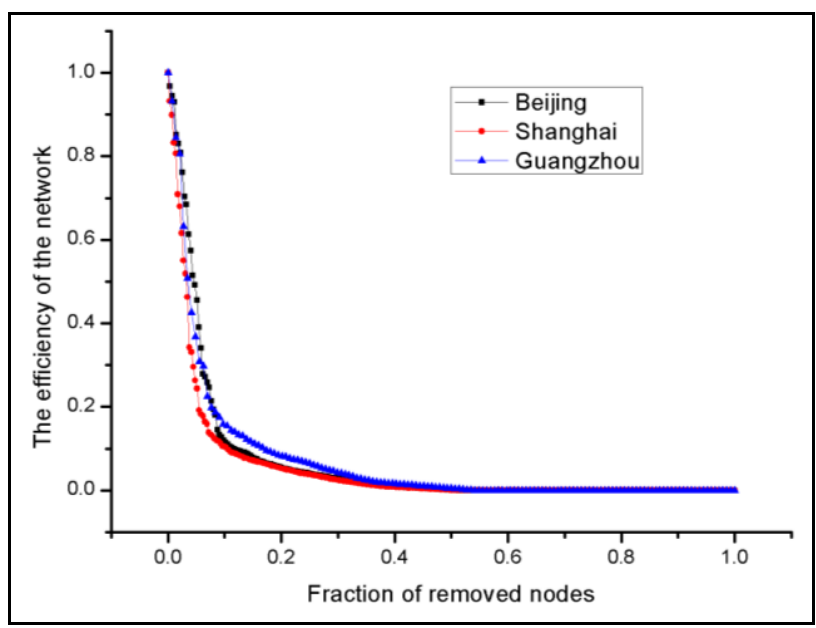

Figure 6. The comparison of network efficiency changes. 
Table 2. The threshold of network efficiency

\begin{tabular}{cc|cc|cc}
\hline \multicolumn{2}{c|}{ Beijing } & \multicolumn{2}{c|}{ Shanghai } & \multicolumn{2}{c}{ Guangzhou } \\
\hline $\begin{array}{c}\text { Fraction of } \\
\text { removed nodes }\end{array}$ & $\begin{array}{c}\text { The efficiency } \\
\text { of the Network }\end{array}$ & $\begin{array}{c}\text { Fraction of } \\
\text { removed nodes }\end{array}$ & $\begin{array}{c}\text { The efficiency } \\
\text { of the Network }\end{array}$ & $\begin{array}{c}\text { Fraction of } \\
\text { removed nodes }\end{array}$ & $\begin{array}{c}\text { The efficiency of } \\
\text { the Network }\end{array}$ \\
\hline 0.50182 & 0.00107 & 0.50172 & 0.00097 & 0.51389 & 0.00314 \\
\hline 0.50545 & 0.00080 & 0.50515 & 0.00073 & 0.52083 & 0.00236 \\
\hline 0.50909 & 0.00053 & 0.50859 & 0.00049 & 0.52778 & 0.00157 \\
\hline 0.51273 & 0.00027 & 0.51203 & 0.00024 & 0.53472 & 0.00079 \\
\hline 0.51636 & 0.00000 & 0.51546 & 0.00000 & 0.54167 & 0.00000 \\
\hline 0.52000 & 0.00000 & 0.51890 & 0.00000 & 0.54861 & 0.00000 \\
\hline
\end{tabular}

\section{Conclusion}

This paper selects urban rail networks of Beijing, Shanghai, Guangzhou as calculation case, gave them a complex network mapping through the L, P Space method and had a static topological analysis using complex network theory, The main conclusions are as follows:

(1). Urban Rail Transit network of Beijing, Shanghai, Guangzhou have the similar network topology, and clustering between sites is extremely small, high clustering between the lines, belong to the random scalefree networks.

(2). The rail network of the three cities are assortative network, i.e. relatively large degree nodes tend to connect relatively large degree nodes. the pearson correlation coefficient of Guangzhou was higher than Beijing and Shanghai. Shows that Urban Rail Transit network of Beijing and Shanghai has been the better development, the Urban Rail Transit network of Guangzhou is still in the growth evolution period.

(3). Network efficiency of urban rail network under the random attack, the maximum degree attack and the maximum betweenness attack, all showed a "waterfall" down, and the "zero threshold" of network efficiency in the three cities is substantially the same, It shows the homogeneity of Urban Rail Transit network topology under different attack patterns.

However, the research object of this paper is mainly for the physical topology of Urban Rail Transit network, and did not take into account the effect of actual line network passengers and railway operation management for the performance of Urban Rail Transit network, whether the performance of Urban Rail Transit network is still exhibit homogeneity under the influence of many factors, this requires further research.

\section{References}

1. National Bureau of Statistics of the People's Republic of China. http://www.stats.gov.cn/

2. S. Boccaletti, V. Latora, Y. Moreno, M. Chavez, \& D.U. Hwang. Complex networks: Structure and dynamics[J]. Physics Reports, 424(4-5):175308(2006).

3. V. Latora, M. Marchiori. Is the Boston subway a small-world network ?[J]. General Information, 314(1):109-113, (2002).

4. P. Angeloudis, D. Fisk. Large subway systems as complex networks[J]. Physica A Statistical Mechanics \& Its Applications, 367:553-558(2006).

5. S. Derrible, C. Kennedy. The complexity and robustness of metro networks[J]. Physica A Statistical Mechanics \& Its Applications, 389(17):3678-3691, (2010).

6. R. Ding, N. Ujang, H.B.Hamid, \& J. Wu. Complex Network Theory Applied to the Growth of Kuala Lumpur's Public Urban Rail Transit Network[J]. Plos One, 10, (2015).

7. Y. Yang, Y. Liu, M. Zhou, F. Li, \& C. Sun. Robustness assessment of Urban Rail Transit based on complex network theory: A case study of the Beijing Subway[J]. Safety Science, 79:149-162, (2015).

8. Y. Liu, Y. Tan. Complexity Modeling and Stability Analysis of Urban Subway Network: Wuhan City Case Study[J]. Procedia - Social and Behavioral Sciences, 96:1611-1621, (2013).

9. J. Zhang, M. Zhao, H. Liu, \& X. Xu. Networked characteristics of the Urban Rail Transit networks[J]. Physica A Statistical Mechanics \& Its Applications, 392(6): 1538-1546, (2013).

10. M.E.J. Newman. 2003b, 'Mixing patterns in networks[J]. Physical Review E, 67(2):241-251, (2003).

11. J. Zhang, X. Xu, L. Hong, S. Wang, \& Q. Fei. Networked analysis of the Shanghai subway network, in China[J]. Physica A Statistical Mechanics \& Its Applications, 390(23):4562-4570, (2010). 